\title{
Evolution of Postgraduate Education in Nuclear Medicine
}

\author{
Sadia Sultana \\ MBBS, DNM (DU), M Sc (Australia), Professor \& Head, Scintigraphy Division \\ Course Director, Postgraduate Education in Nuclear Medicine \\ National Institute of Nuclear Medicine \& Allied Sciences (NINMAS), Email:sadias1964@gmail.com
}

Nuclear Medicine started its journey in Bangladesh in 1962 in the premises of Dhaka Medical College. Gradually other nuclear medicine facilities are established in different parts of the country. At present 15 nuclear medicine establishments are functioning under Bangladesh Atomic Energy Commission (BAEC) including one National Institute of Nuclear Medicine \& Allied Sciences (NINMAS). Three are in Government sectors. One nuclear medicine facility is in combined military hospital and three are in private sectors. Nuclear Medicine is a specialized branch of medicine which is not taught in undergraduate level in Bangladesh. Therefore, initially doctors and physicists were trained on nuclear medicine in abroad. For development of skilled Nuclear Medicine professional, the former Institute of Nuclear Medicine (INM) was established in 1980 (recently named NINMAS) in the then Institute of Post Graduate Medicine and Research (IPGMR) campus. One year diploma course curriculum was approved in 1987. In 1988 the first batch of Diploma in Nuclear Medicine (DNM) was enrolled under DU at this institute. This DNM degree created a great opportunity for young doctors for higher education in Nuclear Medicine in Bangladesh. From 1988 to 1998, a total of 47 students completed DNM including one foreign student. To promote research in the field of Nuclear Medicine, one year course was extended to two years masters program (Master of Philosophy) in 1998 under DU. In the year of 1998, the government converted IPGMR into a Medical University - Bangabandhu Sheikh Mujib Medical University (BSMMU) for expanding the facilities for higher medical education and research in the country. Doctor of Medicine (MD) Nuclear Medicine residency program was started in BSMMU in 2001 but due to some unavoidable circumstances that course did not continue further. However, first batch of MD (Nuclear Medicine) was completed successfully in 2005. In 2010, M Phil Nuclear Medicine has come under BSMMU, the one and only medical university of Bangladesh. From 1998 to 2016, 45 students completed M Phil in Nuclear Medicine and 11 students who enrolled in 2016 are in thesis part now. This is the last batch of M Phil, Nuclear Medicine. BSMMU again introduced its competency-based Residency Program in 2010 and Nuclear Medicine is included in this residency program in 2017. Already 11 students including one foreign student had been enrolled for MD, Nuclear Medicine at NINMAS under BSMMU. Each year, five government students, five private students and one foreign student can be enrolled in MD (Nuclear Medicine) residency program. 